\title{
The Artistic Features of "Zhuo", a Type of Tibetan Dance
}

\author{
Bing $\operatorname{Liu}^{1, *}$ \\ ${ }^{1}$ Mianyang Teachers' College, Mianyang, Sichuan 621000, China \\ "Corresponding author. Email: wdwhysyjzx@163.com
}

\begin{abstract}
The Tibetan "Zhuo" has an ancient history and culture, and its development in Tibetan areas has a long history. It has the reputation of "Chinese living dance fossil". "Zhuo" has a unique ethnic style in the process of development, and policy measures provide Tibetan people with their unique performing arts. In this paper, through the understanding and understanding of "Zhuo", the origin and development of "Zhuo" are studied. On this basis, the differences between it and other dance types are studied to further develop an in-depth understanding of "Zhuo".
\end{abstract}

\section{Keywords: Guozhuang Dance, artistic research, artistic features}

\section{INTRODUCTION}

The Tibetan "Zhuo" is recognized as one of the "National Intangible Cultural Heritage" because "Zhuo" is not only a dance art, but also carries a diverse national culture. In the process of "Zhuo" development, its dance art is more like a carrier of emotions, and it has a unique role in expressing emotions. From a different perspective, its historical value cannot be ignored, so it is necessary to carry out corresponding research on "Zhuo" in modern society.

\section{ORIGIN OF "ZHUO"}

Before studying Guozhuang Dance, it is necessary to study the origin of Guozhuang Dance, which may not be known to many people in China, but has an long history. Guozhuang Dance is a common name for "Zhuo" Dance, which has already appeared in the Tubo period. Guozhuang Dance is a dance that combines the original lyrics of Guozhuang in Qamdo County and some folklore. "Zhuo" is a transliteration of Tibetan. The final stage of "Zhuo" Dance has a close relationship with Tibet's slave society and oath activities. With the continuous transformation of society, forms of singing and dancing gradually emerged. There are three kinds of Tibetan folk dances, namely Guozhuang Dance, Xuanzi Dance and Dagu Dance. Guozhuang Dance is one of these three folk dances. The Guozhuang dance is mainly favored by the Tibetan people in Qamdo, Tibet, and Tibetan Autonomous Prefecture of Garzê, Sichuan. A long time ago, people bought some specialties in one place and sold them to other places. During the trip, they usually stayed in the hotel. This kind of business activity is also called "Guozhuang". In the evening, passengers will prop up the pot with stones on the open space, and then sing and dance together, killing boring time, on the basis of maintaining energetic and maintaining a good mental state in a severe environment. The Guozhuang Dance may have a long history, but the exact time has not yet been confirmed, and it was confirmed by relevant experts in 1993. Inside a big village in Lijiang, the words on the surface of a stele carved with text and portraits was confirmed to be Tibetan. The portrait portrays people's singing posture. The dance posture on the portrait gives people a very religious feeling. Later, the superior got news and some related experts came here to study it. He believed that it is Guozhuang Dance that was portrayed on the stele. And all things are constantly changing, which also has staged differences. The Guozhuang Dance can be divided into different stages of dance at different times, including "Cani" and "Casi", which are the differences between the ancient Guozhuang Dance and the new Guozhuang Dance. The Guozhuang Dance in different periods conveyed the different wishes and feelings of the Tibetan people. Ancient Guozhuang Dance has a strong religious flavor and is also closely related to worship activities. At that time, Guozhuang Dance has its relatively specific content. The dancers are not allowed to change the dance content casually, giving a simple feeling. Therefore, Guozhuang Dance is loved by some old people and religious people. Compared with the ancient Guozhuang, the new Guozhuang dance called "Casi" is more changeable. Most of its contents reflect the bustling scene of labors of farmers and herdsmen, and on this basis, it extols the beauty of laborers. Sometimes some young men and women convey their love through the implicit meaning of the lyrics, which has changed more and become a daily dance that can carry more meaning. 


\section{ARTISTIC CHARACTERISTICS OF GUOZHUANG DANCE}

\section{A. The artistic value of Guozhuang Dance}

The artistic value of the Tibetan "Guozhuang Dance" has a long and continuous history. The cultural heritage of the Chinese nation is very rich. Among them, national arts are diverse and diversify the lives of different ethnic groups. At the same time, the life of the Tibetan people has also been recorded, especially the inheritance of traditional national dances, which has made the world feel the multi-ethnic culture and character. According to the "Introduction to Tibetan Dance", there was a commercial organization called Guozhuang once in Kangting. Such merchants not only purchase and ship local products, but also set up inns. Tibetan merchants often live with the caravans in the inns along the way. At night, they often cook tea in a stone pot in the yard. After tea and dinner, people sing and dance by the fire from time to time to get rid of the day's hard work, and Guozhuang Dance is usually performed by men and women standing separately or mixing into an arc to perform the dance. During the performance, the dancers held hands with each other so that the two teams could get together. The dance form is relatively stable and intuitive. At the same time, the content of Guozhuang Dance can be divided into three parts: the first part is the "overture", the dancers define their position in the overture, and then interlace the dance steps; the second part is the "modeling" of the Guozhuang Dance. The dance style of the Tibetan "Guozhuang Dance" is the basis for generating its rhythm; The third is the expressive "allegro" and the link that most highlights the artistic beauty of the "Guozhuang Dance". This part of the dance content embodies the simple and rugged side of Tibetan dance, and reflects the enthusiasm and passion of people in the dance process. At this time, the dancers sometimes surrounded each other, and sometimes stretched out their arms to fly to the sky, just like eagles spreading their wings in the vast sky. Taking the status quo of "Guozhuang Dance" in Gannan, China as an example, the local Tibetan people's love for "Guozhuang Dance" has been brought into people's daily life. The Guozhuang Dance not only conveys the Tibetans' understanding of the current existence, but also embodies the closeness between people. In the process of being inherited by generation and generation, the culture of dance art continues to accumulate. The longterm cultural accumulation reflects the artistic value of the Tibetan "Guozhuang Dance" of China and also precipitates its artistic value.

\section{B. Analysis of the artistic characteristics of Guozhuang Dance}

When analyzing the artistic characteristics of the Tibetan "Guozhuang Dance", first, it is a must to have a certain understanding of the artistic style of the Tibetan "Guozhuang Dance". The Tibetan "Guozhuang Dance" is one of the indispensable types of dance in China's traditional dance field. Compared with other national dances in China, the artistic characteristics of Tibetan dances are quite unique, which has a great relationship with the living habits and personality characteristics of Tibetan people. Tibetans are good at singing and dancing. In festivals, celebrations, weddings and other activities, people always perform Guozhuang Dance. Guozhuang Dance is a kind of self-entertainment dance. The performances include praising heroic merits, worshipping gods, praising love, celebrating harvest, praising hometown, praising family affection, etc. Generally speaking, there are two styles of Chinese Tibetan dance art: one is that there are few dance movements but various forms; the other is that there are many dance movements and the tempo is extremely fast, which gives people a sense of urgency. "Guozhuang Dance" is a fast and beautiful form of expression, which shows the beauty of dance art in the form of "circle song and dance". From the perspective of essence, from the perspective of contemporary Tibetan dance art research, the Tibetan "Guozhuang Dance" still retains religious elements in the process of transformation, showing a huge dance image. For example, the "circle" is the most commonly used and basic formation in Guozhuang Dance. During the performance, most of the dance movements are performed in the form of "circles", and all movements in the dance are performed in a clockwise direction. The Tibetan masses carried out activities such as turning around, worshipping pagodas, and worshipping temples in a clockwise direction. Therefore, the formation of this feature has a certain relationship with the religious beliefs of the Tibetan people. At the same time, the emotional expression of dance can reflect the Tibetan people's feelings of simple life. On this basis, they express their interpersonal passionate expression elements, while giving Guozhuang Dance a unique artistic feature.

\section{The expression of the artistic value of Guozhuang Dance}

In the process of researching Guozhuang Dance, the artistic value of Tibetan "Guozhuang Dance" is reflected in the following aspects: First, the form of "Guozhuang Dance" is very intuitive, which symbolizes the communication between Tibetan men and women. When people dance "Guozhuang Dance", men and women, hand in hand, under the leadership of one person, in the form of conversation, in the process of "singing and dancing" activities, carry out a communicative activity only with dancing and singing. Guozhuang Dance is a dance with repeating song and dance as its manifestation, and it is very popular among people. The production and development of its dance 
can also reflect its unique artistic charm. The movement of Guozhuang Dance is not only elegant but also simple and decent. Secondly, the expression form of "Guozhuang Dance" reflects the beauty and value of dance art itself. The overall dance of the Tibetan "Guozhuang Dance" is mainly composed of "fast" and "slow" parts. In the process of dancing, people adopt basic movements such as "Youdian Kuatui (slowly bumping and crossing legs)". Most of these movements are performed with the arms and legs of the dancers. Different dance movements are changed by the "swing" and "shake" of the arm. These changes are the embodiments of the core characteristics of the "Guozhuang Dance" art. At the same time, there are certain differences in the performance of Guozhuang Dance in different regions. However, the characteristics of the individual movements are unchanged, and the dancers display the content of the dance in a clockwise formation because they are related to the religious customs of the Tibetan people. The "circle" in the process of dancing is also large and small. In the process of development, the rural Guozhuang Dance is called "Yu Zhuo" in Tibetan. It is divided into two parts: fast and slow. When dancing fast, men and women hold hands, taking turns to sing, talk and raise their legs. After singing the song, everyone shouted "ah". When dancing fast, the dance posture suddenly accelerates with the steps, and the faster people jump during the dance, the higher the interest, and the atmosphere will become pleasant when the dance is completed. In the pastoral area, it is called as "Zhong Zhuo" in Tibetan, and its performance is similar to the Guozhuang Dance in the countryside. The Guozhuang Dance of the monastery is called "Qu Zhuo" in Tibetan. It can be seen from its name that this kind of Guozhuang Dance is solemn and restrained. Generally speaking, singing when holding large Buddhist events or seeing off guests. The dance form is very strict. The dancers are men and wear uniforms, led by a respected dancer. On this basis, the dancers perform the Guozhuang Dance performance with religious significance, which contains its unique artistic value.

\section{Classification of Guozhuang Dance}

In the process of classifying the Guozhuang Dance, according to the occasion of the Guozhuang dance performance, it can be divided into "Big Guozhuang" in large-scale religious sacrificial activities, "Medium Guozhuang" in traditional folk festivals, and "Small Guozhuang" in parties of relatives and friends and so on. The various Guozhuang Dances have different meanings. For its classification, it is divided into the ancient Guozhuang Dance and the new Guozhuang Dance according to the generation time. The ancient Guozhuang Dance has the nature of worship in the development process, and is loved by religious circles and the elderly. The lyrics have a long history, such as the birth of the Lotus Master, the establishment of the
Sangye Temple, and other long-term deeds. For this kind of dance, only specific lyrics can be sung, and they can't be changed casually. The dance moves are slow, intuitive and elegant. The lyrics of new Guozhuang are more flexible and changeable, reflecting various production, labor and business activities. New Guozhuang is very popular among young people. The dance steps of Guozhuang Dance can be divided into two categories: walking dance and turn-around dance. Walking dance starts from one direction and goes to the right, one section with seven steps, starting from the cycle, from slow to fast, and the dance posture is more intuitive and easy to learn, so the number of people studying is very large. Common dances include twoand-a-half steps, six-steps, eight-steps, six-steps plus claps, eight-steps plus claps, and monkey dances. The Tibetan Guozhuang Dance is the most well-known dance in Qamdo. During festivals, celebrations, and weddings, men and women stand on both sides of the square or courtyard, holding hands in a circle, dancing inside, and usually starting with men to sing and women to follow. This song is loud and penetrating. The dancers "talk and dance" and walk along the circle. After the lyrics are sung, people shout "Ya" in unison. And then the rhythm of the song accelerate, people raise their arms high, step forward, stride at the same time, hold up their sleeves, sing and dance, jump and run in the process. Especially for men, the extended arms are like wings spreading like an eagle. The woman moves more, like a phoenix spreading wings when turning her head, which has many characteristics.

Guozhuang Dance is not only diverse in form but also diverse in content. There has been dance called the "wool Guozhuang" reflecting work life and the "celebration Guozhuang" reflecting the wedding. In addition, there are "rabbit Guozhuang" (simulating the jumping of rabbits), "drunk Guozhuang" (imitation of the drunkard's behavior, showing the body's flexible playful movements), etc., and due to different regions, different Guozhuang Dances have their own characteristics. In some areas, the dance music of the Guozhuang Dance is hoarse and rough, like rough waves; the dancing pose is like the wings of an eagle. The Guozhuang Dance music in the Shangri-La region is light and cute. When singing, all the dancers bend their shoulders. The dance is stable. Only when it is near the end, the movement becomes faster, with elegant beauty, and finally the dance is completed in a happy and touching atmosphere. Some Guozhuang Dances have strict procedures from beginning to end: they are divided into Yiwa (Guozhuang overture), Yangzhuo (Zhaofu Guozhuang), Sizhuo (Yingbin Guozhuang), Duizhuo (praising Guozhuang), Zongzhuo (meeting Guozhuang), Zhuzhuo or Zhangzhuo (farewell Guozhuang), Guazhuo (detainment Guozhuang), Zhaxi $\mathrm{Ju}$ (Guozhuang ending song). Each link has its own number or even dozens of tunes. When the dance is 
performed, it must be carried out in accordance with the program. The movement of the dance cannot be changed. It has a unique meaning during the performance. It must be performed step by step. The classification of Guozhuang Dance is diverse, and only by understanding it can one analyze its artistic characteristics.

\section{E. Integration of Guozhuang Dance and modern society}

The combination of the dance culture of Guozhuang Dance and modern society is of great significance in the process of its development. In the process of the transformation of the national dance art, it is necessary to highlight the national style, to enhance national characteristics and enhance its unique national personality on this basis. In addition, the law of national dance is properly researched, and its dance data is taken as the research object, that is, national dance has a strong foundation. And its research must start from its roots. The unique charm of Guozhuang Dance depends on its strong vitality to serve the contemporary national society. The key lies in how to inherit the essence of tradition, how to grasp its essence from vertical to horizontal, and implement a multi-faceted method. In the difficult history of national dance, it is always in the process of production and creation, and the mission of each generation is to create something that was not in the past. As long as working tirelessly, people can create a new "traditional" Guozhuang Dance. If the cultural and sports workers and social counselors can control the style and rhythm of Guozhuang Dance, and integrate the characteristics and charm of Tibetan dance with modern society, they can achieve the civilianization of national dance, on the basis of which to enhance their sustainable development, providing impetus for its development. The knowledge gained in contemporary society has reached a certain stage. It is necessary to expand the knowledge field and integrate it on this basis. In order to absorb more nutrients to strengthen the Tibetan folk dance, after absorbing enough nutrients, it contains the essence of traditional dance. The inheritance can be carried out on this basis. However, Guozhuang Dance is a group dance. The participating groups are the public and it can also be called as social dance. The original meaning of "social dance" refers to the dance of non-professional dancers and the dance of non-stage art. According to these two characteristics, Guozhuang Dance can become a "social dance", which is not based on the premise of technicality and standardization, but on the basis of its functionality. In the process, it does not reflect life but participates in life, thereby enhancing the life of Guozhuang Dance and further embodying its artistic characteristics.

\section{CONCLUSION}

All in all, Guozhuang Dance is the creation of the perfect life and the spiritual world of the people. It plays an important role in improving the overall quality of a nation and the evolution of culture. On this basis, Guozhuang Dance reflects the diligent and strong national character of the Tibetan people. At the same time, it reflects the desire of the Tibetan people for beauty and the wisdom and aesthetic feelings of their people, with a strong sense of life. And Guozhuang is an indispensable part of the Chinese nation's art palace, and it is a must to have a sufficient understanding of its value.

\section{References}

[1] Shi Yan, Artistic Features of Tibetan Guozhuang Dance [J]. Big Stage, 2014, (8): 221-222. (in Chinese)

[2] Mo Shangyang, The Artistic Characteristics of Tibetan Guozhuang Dance [D]. Sichuan: Sichuan Normal University, 2012. (in Chinese)

[3] Zhang Lina, An Analysis of Origin and Art Characteristic of Guozhuang Tibet Dance [J]. Journal of Hubei Correspondence University, 2016, (4): 190-191, 194. (in Chinese)

[4] Zhaxi Jiangcuo, Tibetan Traditional Dance Course [M] Shanghai Music Publishing House, 2015: 8-10. (in Chinese)

[5] Yang Xifan. Ethnic Minority Music and Dance Culture in the "Tibet-Yi Corridor"[J]. Art Studies, 2007, (1): 93. (in Chinese)

[6] Nima Ciren. Traditional Tibetan Music and Dance - An Interview of the Famous Tibetan Artist and Professor Sonam Dajie Shrukhang $[\mathrm{J}]$. Journal of Tibet University (Chinese Edition),2007(03):1. (in Chinese) 